\title{
The evolution of dorsal-ventral patterning mechanisms in insects
}

\author{
Jeremy A. Lynch and Siegfried Roth ${ }^{1}$ \\ Institute for Developmental Biology, University of Cologne, 50674 Cologne, Germany
}

The gene regulatory network (GRN) underpinning dorsal-ventral (DV) patterning of the Drosophila embryo is among the most thoroughly understood GRNs, making it an ideal system for comparative studies seeking to understand the evolution of development. With the emergence of widely applicable techniques for testing gene function, species with sequenced genomes, and multiple tractable species with diverse developmental modes, a phylogenetically broad and molecularly deep understanding of the evolution of DV axis formation in insects is feasible. Here, we review recent progress made in this field, compare our emerging molecular understanding to classical embryological experiments, and suggest future directions of inquiry.

Bilaterally symmetric animals are defined by the presence of two orthogonal axes of symmetry: the anterior-posterior (AP) axis, running parallel to the gut from mouth to anus, and, perpendicular to this, the dorsal-ventral (DV) axis. Despite the wide variety of disparate modes of embryogenesis and final forms found among the animals, some highly conserved signaling pathways and transcription factor networks have been found to govern axis formation in the majority of animals. For example, recent findings suggest that Wnt signaling plays a conserved role in establishing the AP axis of most bilaterian animals by controlling the expression of the Hox cluster genes (Petersen and Reddien 2009). In addition, the use of graded BMP signaling is broadly conserved in both setting up the DV axis in early embryogenesis (De Robertis 2008) and DV patterning of the ectoderm in later development (Mizutani and Bier 2008). Despite these conserved molecular principles of many aspects of axis formation, some major transitions in strategies for axial patterning have occurred in animal evolution. For example, Wnt signaling is not involved in AP axis establishment in Drosophila, while a novel gene, bicoid, performs the major patterning role. Similarly, the role of BMP signaling in establishing DV polarity is strongly reduced in Drosophila, where Toll signaling (which has a highly conserved, ancestral role in innate immunity) is responsible for generating the vast majority of DV patterning

[Keywords: BMP signaling; dorsal; Drosophila; Nasonia; Toll signaling; Tribolium]

${ }^{1}$ Corresponding author.

E-MAIL siegfried.roth@uni-koeln.de; FAX 49-0221-4705164.

Article is online at http://www.genesdev.org/cgi/doi/10.1101/gad.2010711. information. Thus, understanding the evolution of axial patterning can provide valuable insights into how conserved regulatory networks adapt to novel patterning environments, and how evolutionary novelties can be incorporated into an established patterning system.

DV patterning in insects provides an ideal system for studying how developmental evolutionary change affects the interplay between conserved and nonconserved mechanisms. The advantages of insects as a model clade include the following: (1) They represent a spectacularly diverse assemblage of species, and thus a large number of potential sampling points are available with which the evolutionary history of insect DV patterning mechanisms can be reconstructed. (2) Many radical transitions in modes of embryogenesis (such as polyembryony and viviparity) have arisen multiple times in insects, allowing the exploration of how the DV patterning network adapts in the face of evolutionary novelties. (3) Many insect species have had their full genomes sequenced (Weinstock et al. 2006; Richards et al. 2008; The International Aphid Genomics Consortium 2010; Werren et al. 2010), making the identification of key components and targets of the DV patterning network more straightforward and efficient. (4) Crucially, techniques to test gene function-such as germline transformation (Berghammer et al. 1999; Peloquin et al. 2000; Tamura et al. 2000; Pavlopoulos et al. 2004; Nakamura et al. 2010) and parental RNAi (pRNAi), where gene function can be knocked down in embryos by injecting dsRNA into pupal or adult females (Hughes and Kaufman 2000; Bucher et al. 2002; Miyawaki et al. 2004; Lynch and Desplan 2006; Ohde et al. 2009/—are widely applicable throughout the insects (see Fig. 1), which allows a deep understanding of gene interactions that is complementary to the opportunities for broad phylogenetic sampling of insect DV patterning mechanisms. (5) Finally, the almost complete understanding of Drosophila DV axis patterning serves as a powerful source of comparative information and hypothesis generation.

\section{Setting up the DV axis in Drosophila}

Since the Drosophila DV patterning gene regulatory network (GRN) is the gold standard to which other insects will be compared, crucial aspects of the functioning of this network are described here (for more in depth review, see Roth 2003; Moussian and Roth 2005; Hong et al. 2008; 


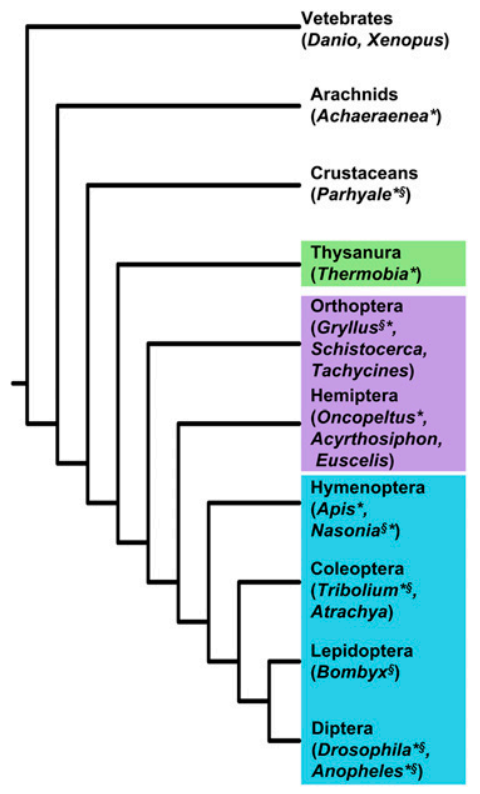

Figure 1. Phylogenetic tree of species discussed in the text. The green box denotes ametabolous insects (hatchlings similarly proportioned to mature adults), the purple box denotes hemimetabolous insects (hatchlings lack functional reproductive organs and some adult structures such as wings), and the blue box denotes holometabolous insects (hatchlings are highly reduced larvae, and undergo complete metamorphosis in a pupal stage). ( $\left.{ }^{\star}\right)$ Availability of $\mathrm{RNAi}_{;}\left({ }^{\S}\right)$ availability of germline transformation.

Roth and Lynch 2009). The cascade of molecular interactions that lead to the establishment of cell fates along the DV axis of the Drosophila embryo begins with a symmetry-breaking event in the ovary, when the oocyte nucleus moves to a cortical position that is asymmetric with regard to the short axis of the egg chamber (Roth and Lynch 2009). mRNA for gurken (grk) is localized around the oocyte nucleus and translated, and its protein (encoding a tgf- $\alpha$-like EGF ligand) is secreted to the overlying somatic follicle cells, where it activates the EGF receptor (EGFR) (Fig. 2A; Schupbach and Roth 1994). The main target of Grk signaling relevant to DV axis patterning is pipe, whose expression is repressed in the dorsal follicle cells in response to EGFR activation (Fig. 2A; Sen et al. 1998).

Pipe encodes a sulfotransferase whose targets were identified recently as vitelline membrane resident proteins (Zhang et al. 2009a). The ventral restriction of Pipe function is important to localize the activity of a serine protease cascade (Fig. 2B)-likely through interactions of its modified substrate with the Snake and Easter proteases (Cho et al. 2010) - to the ventral region of the perivitelline space. This protease cascade leads to the cleavage and activation of the Toll ligand Spätzle $(\mathrm{Spz})$ in the ventral portion of the perivitelline space (Fig. 2B; Moussian and Roth 2005).

After the egg is laid, cleaved Spz protein binds and activates the maternally supplied Toll receptor present on the plasma membrane of the embryo. Activation of Toll leads to the phosphorylation and degradation of the ІкB homolog Cactus (Cact) (Fig. 2C), which, in the absence of signaling, binds the NF- $\mathrm{B}$ transcription factor Dorsal and sequesters it in the cytoplasm. The phosphorylation of Cact allows the translocation of Dorsal to the nucleus, where it binds specific DNA sequences and regulates the transcription of numerous genes (Fig. 2C; Moussian and
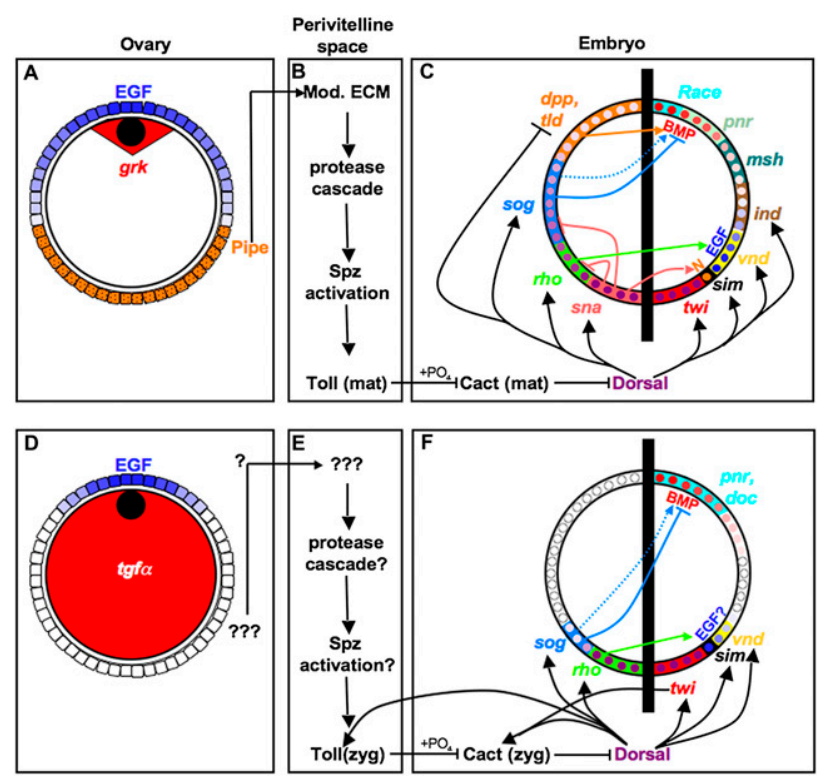

Figure 2. Patterning the DV axis in Drosophila and Tribolium. $(A-C)$ DV patterning cascade of Drosophila. $(A)$ In the ovary, localization of grk mRNA (red) around the oocyte nucleus leads to the activation of the EGF pathway (blue) in the follicle cells, restricting pipe expression to the ventral follicle cells. $(B)$ In the perivitelline space, ECM components modified by pipe lead to the localized activation of a protease cascade and, ultimately, to the activation of Spz, which binds and activates the Toll receptor. $(C)$ In the embryo, Toll activation leads to phosphorylation and degradation of Cact, allowing nuclear translocation of Dorsal in a ventral-to-dorsal gradient (purple circles) where it regulates the expression of target genes. Components of the BMP ( $s o g, d p p$, and tld) and EGF (rho) signaling pathways and a regulator of Notch (N) signaling (sna) activated by different thresholds of Dorsal concentration are shown on the left side of the split embryonic cross-section. On the right side, tissue-specific target genes that integrate input from one or more of these pathways with that of Dorsal concentration are shown. $(D-F)$ DV patterning cascade of Tribolium. $(D)$ Like Drosophila, EGF signaling is activated in association with the position of the oocyte nucleus. However, mRNA for the ligand $(\operatorname{tgf} \alpha)$ is not localized. Targets of EGF signaling have not yet been identified in the Tribolium ovary, and it is not clear how patterning information is transmitted to the embryo. (E) Preliminary evidence indicates that the protease cascade and Spz are required for Toll activation, but how or whether these events are restricted to the ventral perivitelline space is not known (indicated by ? ??). (F) Unlike Drosophila, there is considerable feedback regulation at the zygotic level in the Tribolium DV patterning system. As in $C$, the left half of the cross-section illustrates the expression of signaling pathway components just before gastrulation, while the right half shows the expression of target genes of Dorsal and/or downstream signaling pathways. For EGF signaling, the pathway has been shown to be activated laterally, but it has not been demonstrated that it has direct input into vnd or sim expression. 
Roth 2005). Toll activation occurs in a gradient emanating from the ventral midline, leading to a corresponding gradient of Dorsal nuclear uptake. This gradient is stable throughout the 14th synchronous nuclear division cycle, when most embryonic patterning takes place, and shows a similar shape with lower amplitude during the preceding five syncytial divisions (Kanodia et al. 2009; Liberman et al. 2009).

The graded pattern of nuclear Dorsal uptake is not simply a readout of the ventral pipe expression pattern in the ovarian follicle cells, because the latter is expressed uniformly in its domain (Fig. 2A; Roth 2003). This paradox is deepened by mutations-such as those affecting grk and other EGF signaling components - that result in the expansion of the pipe expression domain. Instead of simply expanding, the Dorsal gradient splits, resulting in two peaks of ventralizing activity in the embryo (Moussian and Roth 2005). Thus, there must be dynamic, self-regulatory mechanisms that allow the transformation of the uniform pipe domain in the ovary into a graded domain of Dorsal nuclear uptake in the embryo. This self-regulation appears to occur at the level of Spz activation (Morisato 2001), but its exact nature is not yet clear. It is also interesting to note that recently it has been suggested that there might be a source of DV polarity information in addition to pipe, since embryos derived from egg chambers expressing pipe ubiquitously in the follicle cells still show some degree of polarity along the DV axis (Zhang et al. 2009b). This may be related to the earlier finding that BMP signaling molecules expressed during oogenesis might have a direct input into Toll signaling in the embryo (Araujo and Bier 2000).

\section{Interpretation of the Dorsal gradient}

The establishment of the nuclear gradient of Dorsal is the pivotal event in DV axis formation in Drosophila, because all cell fates along this axis depend directly or indirectly on Dorsal for their proper regulation. Dorsal acts as a morphogen, activating and repressing target genes in a concentration-dependent manner (Fig. 2C). The differential sensitivities of Dorsal target genes is reflected, in part, by the affinities and arrangement of Dorsal-binding sites in their respective enhancers, such that enhancers of ventrally expressed genes possess low-affinity sites, while those that respond to the low levels of nuclear Dorsal on the dorsolateral side of the embryo are composed of high-affinity binding sites (Stathopoulos and Levine 2004; Hong et al. 2008).

The expression of target genes is not, however, completely specified by the Dorsal-binding sites in their enhancers. Dorsal cooperates with other maternal factorssuch as Zelda, basic helix-loop-helix (bHLH) proteins, and at least one unidentified factor-to achieve the most dorsally extending expression domains (Crocker et al. 2008; Liberman and Stathopoulos 2009). In addition, Dorsal cooperates with its ventral targets twist (twi) and snail (sna) to pattern the mesoderm and ventral neurectoderm. Finally, Dorsal regulates components of other signal transduction pathways (Hong et al. 2008). The most important of these for establishing the initial polarity of the embryo are the EGF and BMP pathways (Fig. 2C). EGF signaling is activated in the neurogenic ectoderm as a result of the lateral expression of the Dorsal target rhomboid (rho). The resulting gradient of EGFR activation helps to set and refine the borders of genes patterning the neurogenic ectoderm (Schweitzer et al. 1995; Gabay et al. 1996; Von Ohlen and Doe 2000; Hong et al. 2008).

Dorsal also has a major role in regulating components of the BMP signaling pathway. It activates a broad lateral domain of the BMP inhibitor short gastrulation (sog) and represses the expression of the BMP2/4-like ligand decapentaplegic (dpp) and the metalloprotease tolloid (tld) such that they are restricted to the dorsal side of the embryo (Fig. 2C; Stathopoulos and Levine 2005). This arrangement of pathway component expression leads initially to a gradient of BMP activation that patterns the dorsal side of the embryo. This gradient later resolves into a sharp peak at the dorsal midline as a result of complex proteinprotein interactions among BMP components $\left(\mathrm{O}^{\prime}\right.$ Connor et al. 2006) and transcriptional feedback (Wang and Ferguson 2005).

\section{Drosophila uses highly derived modes of embryogenesis and oogenesis}

Although the DV patterning network of Drosophila is understood in great detail, and thus serves as a critical basis for comparative studies, the fly exhibits highly derived features and is not typical of insects in several respects. Drosophila undergoes what is termed long-germ embryogenesis, where all segmental (AP) and germ layer (DV) fates are represented in a coordinate system on the surface of the blastula stage embryo. This mode of embryogenesis allows for the rapid and nearly simultaneous assignment of DV fates to cells representing the entire segmental extent of the future larva and adult fly (Fig. 3D; Davis and Patel 2002). Long-germ embryogenesis is found only among holometabolous insects, where the consensus opinion is that this mode has been derived multiple times independently.

This is in contrast to the ancestral short-germ mode of embryogenesis, found in all hemimetabolous insects and some holometabolous insects such as the beetle Tribolium castaneum. In this type of embryogenesis, the embryonic primordium is often (but not always) (Fig. 3E) small in comparison with the entire egg surface; only some of the anterior segments (often the head and thoracic segments) are represented at the blastula stage, while the remainder are generated and patterned progressively from a posterior growth zone (Fig. 3E,F; Davis and Patel 2002). Thus, short germ embryogenesis requires two phases of DV patterning: one operating at the blastula stage, where a Drosophilalike system could be used, and one operating in the growth zone after gastrulation, which has no clear counterpart in the fly. Furthermore, it is not clear whether a mechanism that is dependent on maternally established, global, DV egg polarity is necessary-or would even be effective-in species such as Schistocerca americana, where the embryo primordium is very small in relation to the large, yolky, egg (Dearden and Akam 2001). 


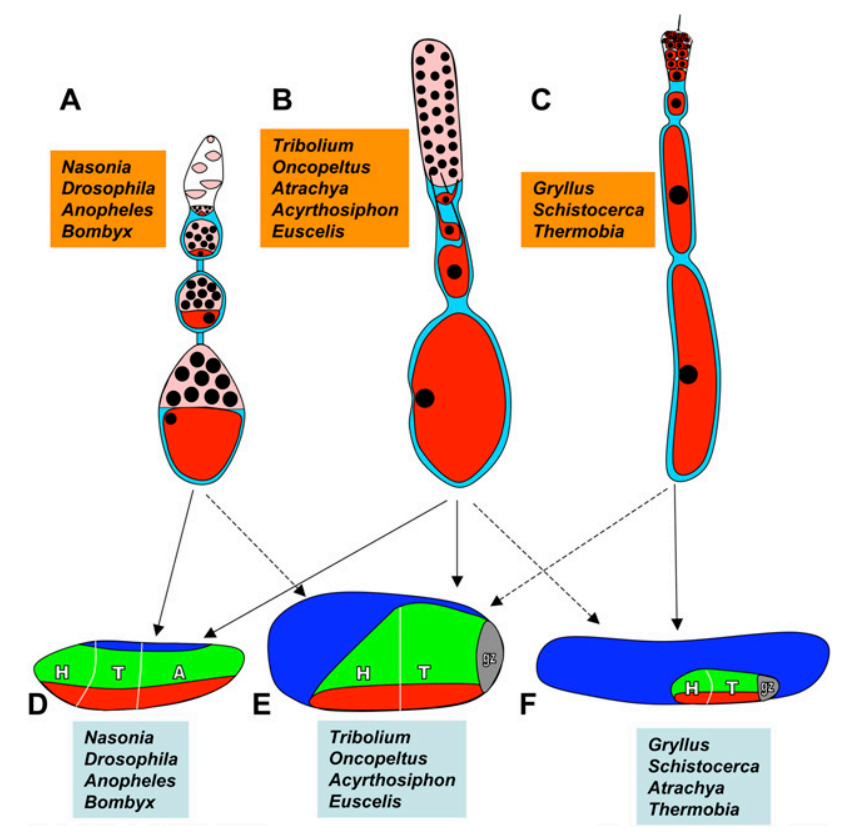

Figure 3. Comparison of the main ovary and embryo types among insects. $(A-C)$ Structure of ovarioles representing the three main types of insect oogenesis: polytrophic meroistic $(A)$, telotrophic meroistic $(B)$, and panoistic $(C)$. Light blue represents the somatic follicle cell layer, red denotes oocytes, and pink marks nurse cells. Orange text boxes indicate species mentioned in the text associated with each type of oogenesis. $(D-F)$ Different arrangements of cell fates in pregastrulation blastoderm stage embryos. (D) A long-germ embryo. (E) A short-germ embryo with a large germ rudiment. $(F)$ A short-germ embryo with a germ rudiment small in relation to the egg. Blue represents extraembryonic membranes, green indicates ectoderm, and red denotes mesoderm. Light-blue boxes indicate taxa mentioned in the text associated with each type of embryogenesis. Full arrows indicate primary associations between ovary and embryo type, and dashed arrows indicate rare associations.

This question in regard to the nature and amount of maternally provided DV patterning information is related to another major difference between Drosophila and the ancestral system in insects: its mode of oogenesis. The basic unit of the Drosophila ovary is the egg chamber, which is composed of three general cell types: (1) somatically derived follicle cells, which form a monolayer on the surface of the egg chamber; (2) the germline-derived oocyte, which gives rise to the egg and embryo; and (3) the nurse cells, also of germline origin, which are specialized for synthesizing the large amounts of mRNA and cytoplasmic components required to support the rapid development of the fly embryo. These nurse cells are sister cells of the oocyte, arising by incomplete cytoplasmic divisions of a germline stem cell. This is termed polytrophic meroistic oogenesis (Büning 1994); it is found mainly in holometabolous insects, and is associated (but not completely correlated) with long-germ embryogenesis (Fig. 3A; Roth 2004).

Another type of oogenesis that includes germlinederived nurse cells is termed telotrophic meroistic oogen- esis. In this mode, the nurse cells are located at the anterior tip of each ovariole, serve as a common source for mRNAs and cytoplasmic components to all oocytes, and have no clonal relationship to the oocytes. Again, the germline-derived cells are surrounded by a monolayer of somatic follicle cells. This type of oogenesis is found in both hemimetabolous (primarily the Paraneoptera, the sister group to the Holometabola) and holometabolous insects, and is associated primarily with short-germ embryogenesis, but it also can support long-germ development (Fig. 3B; Büning 1994).

Finally, the ancestral mode of oogenesis for the insects is termed panoistic. In this mode, all germline cells become oocytes, surrounded again by a somatic follicular epithelium, and all maternal mRNA and cytoplasmic components required for embryonic development must be synthesized by the oocyte nucleus (Fig. 3C; Büning 1994). This mode is associated almost exclusively with short-germ embryogenesis.

\section{Major themes in the evolution of DV patterning of insects}

Given the diversity in modes of embryogenesis and oogenesis found among insects, the novelty of using Toll signaling as a basis for embryonic patterning, and the long evolutionary history of insects that can be sampled functionally, a number of general themes have emerged to direct a research program aimed at understanding the evolution of the DV patterning regulatory network within this diverse animal clade: (1) How is positional information generated during oogenesis and transmitted to the embryo in insects with divergent modes of embryogenesis and oogenesis? (2) How are the asymmetries translated into patterning gradients in the embryo in these systems? (3) How does enhancer logic change in response to different gradient/embryo types? (4) How do growth zone and blastoderm patterning differ-at both the molecular and embryological level? (5) When did the use of Toll to pattern the DV axis of the embryo originate among insects or arthropods, and what was the ancestral strategy that it replaced?

\section{Generating maternal cues for DV patterning in insects}

Insects are unusual among animals in that their eggs very often show clear bilateral symmetry (presaging the future $\mathrm{AP}$ and DV axes of the embryo) when they are laid, indicating that symmetry along both axes is broken during oogenesis in this clade. The potential role of the position of the oocyte nucleus in combination with signaling from the oocyte to the follicle cells in establishing the DV axis was already hypothesized in the middle of the last century (Netzel 1965, 1968), and the discovery and characterization of grk provided a molecular basis for how this symmetrybreaking event could be effected (Neuman-Silberberg and Schuepbach 1993). However, it was not clear whether the Drosophila system could be applied to other insects, since clear grk orthologs had not been found outside of the higher diptera. With the sequencing of several complete insect 
genomes, it became clear that grk was a rapidly evolving member of a group of three tgf- $\alpha$-like ligand paralogs in Drosophila, and that, ancestrally, there was a single ortho$\log$ (Lynch et al. 2010).

To test the idea that the EGF pathway had an ancestral role in establishing DV polarity among insects, components of the EGF pathway were examined in insects representing a broad sampling of insect phylogeny: the cricket Gryllus, the wasp Nasonia, and the beetle Tribolium. In all three species, mRNA for the single Tgf- $\alpha$-like ligand is expressed in the germline, mRNA for the EGF receptor is expressed in the follicle cells, and activation of the pathway occurs asymmetrically in the follicular epithelium in association with the position of the oocyte nucleus (Lynch et al. 2010).

When the EGF signaling pathway is disrupted by pRNAi against some of its components (e.g., the ligand or EGFR) dramatic disruption of DV polarity in all three tested organisms is observed, showing that EGF-mediated germline-to-soma signaling is an ancient strategy for establishing DV patterning among insects (Lynch et al. 2010). In Gryllus, pRNAi against $G b-\operatorname{tg} f \alpha$ leads to a loss of DV asymmetry of the oocyte, which has been shown to be strongly correlated with the future DV axis of the embryo (Netzel 1968). In Nasonia and Tribolium, severe effects on embryonic DV patterning were observed, and the range of phenotypes are reminiscent of the range given by grk alleles of different strengths (Roth and Schupbach 1994). These phenotypes included simple broadening and partial duplications of ventral and lateral marker gene expression. This latter phenotype indicates that selfregulation downstream from maternally generated asymmetries is a conserved property of insect DV patterning systems. In addition, since the Dorsal gradient of Tribolium follows the same patterns of disruption as the ventral marker genes, maternal EGF signaling has a conserved role upstream of Toll signaling to constrain the generation of the ventralizing gradient to a single peak parallel to the AP axis of the embryo.

While these results show that the role of maternal EGF signaling in establishing DV polarity is broadly conserved in insects, a number of outstanding questions remain about how the maternally generated DV asymmetries in the oocyte are translated into embryonic patterning information. One major missing component is pipe, which showed no phenotype with pRNAi and had no detectable asymmetry in its expression in any of the three examined species (Lynch et al. 2010). Thus, a different mode of translating the asymmetric activation of EGF signaling in the follicle cells into embryonic patterning information is likely present in insects outside of Drosophila (Fig. 2D). It will be interesting to see whether this mechanism is related to the additional source of DV asymmetry proposed to exist in Drosophila (Zhang et al. 2009b).

In Nasonia, Nv-tgf $\alpha$ mRNA is localized to the oocyte nucleus, similar to grk in Drosophila, as well as in a stripe extending to the posterior pole. This apparently convergent use of localized mRNA in Drosophila and Nasonia indicates that mRNA localization is a common solution to the problem of how to provide a robust source of posi- tional information along the DV axis required for the demands of the long-germ mode of embryogenesis. Interestingly, localized mRNA seems to have a similar status in patterning the AP axis (Lynch et al. 2006; Brent et al. 2007; Olesnicky and Desplan 2007).

In contrast, $\operatorname{tg} f \alpha$ mRNA is not localized in either Tribolium (Fig. 2D) or Gryllus, and EGF signaling is activated in the follicle cells in a restricted, circular gradient emanating from the region directly overlying the oocyte nucleus in both species (Fig. 2D; Lynch et al. 2010). This raises a further question as to how this apparently limited amount of asymmetric information is transformed into a gradient of high enough precision to efficiently specify cell fates along the DV axis. Theoretical modeling has shown that self-regulatory systems are capable of transforming weak asymmetries such as these into robust patterns (Meinhardt 1989). pRNAi against tgf $\alpha$ in Tribolium and Nasonia indicated that the DV patterning has self-regulatory properties, and selfregulation may be an ancestral feature of insect patterning systems.

So far, it is not known whether ligand activation is conserved as the level at which self-regulation occurs (Fig. 2E), but work in Tribolium has shown that there is an additional strategy to achieve self-regulation in DV patterning systems.

\section{Transforming maternal asymmetries into patterning gradients}

The Drosophila Dorsal gradient is stable over time, and its effects can be observed quite far from its peak at the ventral midline. Thus, it was surprising when it was discovered that the Tribolium Dorsal (Tc-Dorsal) gradient is dynamic over developmental time: In early embryos, it is extremely broad, then becomes progressively restricted, until it finally disappears just before gastrulation (Chen et al. 2000). Recently, it was shown that this dynamic Tribolium Dorsal gradient arises as a result of feedback loops involving its zygotic target genes (da Fonseca et al. 2008). Both the upstream activating receptor Toll and the repressor cact are expressed dynamically in a pattern mostly following that of nuclear Dorsal and are likely direct Dorsal targets (Fig. 2F). Furthermore, the ventral expression of $t w i$, another Dorsal target, is required for the maintenance of cact expression at the ventral midline (Fig. 2F), leading to the final extinction of Dorsal nuclear localization (da Fonseca et al. 2008). The use of zygotic feedback loops to generate the dynamic Tribolium Dorsal gradient stands in stark contrast to the fly Dorsal gradient, which is strictly hierarchical and is established by the interactions of maternally supplied factors. However, a remnant of the Tribolium system seems to remain in Drosophila in the form of a ventral domain of Dorsaldependent cact expression that has been uncovered recently in an analysis of genome-wide transcription factor binding (Sandmann et al. 2007).

Results of Tc-tgf $\alpha$ pRNAi experiments have shown that the Tc-Dorsal-centered feedback loops are able to function in the absence of strong maternal EGF input (Lynch et al. 2010). The irregularities of DV patterning observed 
under these conditions suggest that the system is able to enhance stochastic fluctuations present in initial concentrations of signaling components. This is expected for patterning systems able to sense and amplify weak starting asymmetries (Meinhardt 2004).

Like its fly counterpart, Tribolium Dorsal does not act alone in establishing cell fates along the DV axis of the embryo. Some evidence exists indicating that, as in Drosophila, components of the EGF pathway are downstream from Dorsal in Tribolium (Fig. 2F; Wheeler et al. 2005; Rousso et al. 2010). In addition, Tc-Dorsal activates the diffusible BMP inhibitor short gastrulation (Tc-sog) in a broad ventral domain (Fig. 2F; da Fonseca et al. 2008). Tc-sog has two related functions in early Tribolium embryogenesis: (1) It inhibits BMP signaling on the ventral side, where it binds ubiquitous Tc-Dpp, and (2) it transports bound Tc-Dpp to the dorsal side, where it is released and activates BMP receptors, resulting in a broad peak of BMP activation on the dorsal surface of the embryo (Fig. $2 \mathrm{~F})$. This domain of BMP activation is required to pattern the dorsal portions of the extraembryonic membranes (serosa and amnion) and the dorsal ectoderm (van der Zee et al. 2006). In contrast to Drosophila, Tc-dpp expression is not regulated by the Tc-Dorsal gradient.

Due to the different nature of the Tribolium early embryo fate map, where the head primordium is both an anterior and ventral fate at the blastoderm stage, perturbation of BMP signaling has major effects on anterior embryonic fates. Knockdown of $T c-d p p$ results in a ventralization of the embryo, and thus an expansion of the head anlage, while knockdown of Tc-sog results in ectopic BMP activation along the border between the extraembryonic and embryonic primordia, leading to the deletion of head segments (van der Zee et al. 2006). This cross-talk between the AP and DV axis is in contrast to the general independence of the two axes in Drosophila, while such cross-talk is typical of vertebrate early embryo patterning (De Robertis 2008).

In Drosophila, the formation and refinement of the dorsal BMP activity gradient is the result of a complex network of protein-protein interactions in the extracellular space (see above). Similar interactions are also required in Tribolium, but on the whole the network employed in the early embryo appears to be much simpler in the beetle compared with the fly (da Fonseca et al. 2010). In Drosophila, twisted gastrulation (tsg) has two additional paralogs in the genome (crossveinless and shrew), and tld has its paralog (tolkin), while in Tribolium, there are single orthologs of each of the major components. In addition, only $T c-d p p$ and its type I receptor, $T c-t k v$, are required during blastoderm patterning, while in Drosophila the additional ligand screw and type I receptor saxophone are required for full BMP activation (da Fonseca et al. 2010). Interestingly, these latter two genes are required for DV patterning of the segments arising from the growth zone after gastrulation. A further novel property of BMP signaling in the early Tribolium embryo is that the function of Tc-tsg is absolutely required for BMP activation. This differs significantly from what has been found in both Drosophila, where tsg and shrew are required only for peak levels of BMP activation, and vertebrates, where tsglike genes have both positive and negative effects on BMP signaling (da Fonseca et al. 2010). It will be interesting to see whether the absolute dependence of BMP signaling on Tc-tsg represents the ancestral state, or whether it is a unique property of the Tribolium system.

\section{Sensing and interpreting patterning gradients}

Once patterning gradients have been established in the early insect embryo, downstream effector genes of different cell fates must be activated in their proper domains to establish different cell fates. The cis-regulatory logic required for proper expression of genes in different domains along the DV axis of the Drosophila embryo is now well described due to the development of techniques for wholegenome discovery-and computational prediction-of transcription factor-binding sites (Markstein et al. 2004; Papatsenko 2007; Sandmann et al. 2007; Zeitlinger et al. 2007). Understanding how cis-regulatory modules (CRMs) of DV-regulated genes change in response to different modes of early development and divergent dynamics of patterning gradient formation, such as those described above, will provide a major step forward in understanding how GRNs for developmental processes can evolve.

An example from the genus Drosophila shows that adaptations to different embryonic patterning conditions can happen over a relatively short evolutionary time. CRMs of genes expressed in the ventral neurectoderm (e.g., vnd, rho, and brk) of the Drosophila melanogaster embryo are characterized by the shared presence and specific arrangement of Dorsal, Twi, Sna, and other transcription factor-binding sites (Erives and Levine 2004). Despite the general absence of significant conservation of noncoding regions at the nucleotide level between $D$. melanogaster and its congeners, Drosophila pseudoobscura and Drosophila virilis, CRMs for ventral neuroectoderm genes were detected in the latter two species based on the same criteria used to detect this class of enhancer within the D. melanogaster genome (Crocker et al. 2008). When the CRMs from D. pseudoobscura and $D$. virilis ventral neuroectoderm genes were used to drive transcription in $D$. melanogaster, the $D$. virilis CRMs consistently drove expression in a domain broader than the native $D$. melanogaster cognate gene, while the D. pseudoobscura enhancers drove consistently narrower patterns (Crocker et al. 2008). These results indicate that there have been coordinated changes in the ventral neurectoderm enhancers specific to each of these lineages. These changes correlate in a logical way with differences in the extent of the Dorsal gradient among the examined species, but influences of Dorsal protein sequence evolution or genome architecture could not be excluded.

Another study on DV CRM evolution within the Drosophila genus focused on enhancers for sog. sog is among the most sensitive Dorsal targets, and is expressed in a broad lateral domain that is conserved from $D$. virilis to $D$. melanogaster ( $\sim 40$ million years of independent evolution) (Liberman and Stathopoulos 2009). Drosophila 
sog enhancers are characterized by the presence of highaffinity Dorsal-binding sites, very few of which are conserved in sequence or position, but these are not sufficient to drive sog expression in the most dorsal region of its domain. Rather, sog requires Dorsal cooperation with the ubiquitous general activator Zelda to achieve its full expression (Liberman and Stathopoulos 2009). Liberman and Stathopoulos (2009) showed that other genes expressed in patterns similar to that of sog also require input from ubiquitous activators, but that factors other than Zelda (such as STAT) can provide the cooperating input. Using these insights, synthetic enhancers composed of different combinations of Dorsal- and STAT- or Zeldabinding sites could be created to drive expression in soglike domains (Liberman and Stathopoulos 2009). These results demonstrate the flexibility of strategies available to evolution to drive gene expression in particular domains, and provide valuable groundwork for dissecting DV CRMs in other insects.

Sampling from a broader phylogenetic range of insects has shown both that much DV CRM logic has been conserved over hundreds of millions of years of independent evolution, and that lineage-specific alterations of the inputs to these CRMs have also occurred. In Drosophila, the mesectoderm marker single-minded (sim) resolves into single-cell-wide stripes flanking the mesoderm primordium. Its CRM mediates positive transcriptional inputs from Dorsal, Twi, and Supressor of Hairless $[\mathrm{Su}(\mathrm{H})$; an effector of the Notch (N) pathway], and negative input from Sna. In the bee Apis melifera, sim is also initially expressed in stripes flanking the presumptive mesoderm, but the pattern differs from the fly in that the Apis sim stripes are much broader (up to six cells wide) (Zinzen et al. 2006). A putative Apis sim CRM was identified by the presence of clustered Dorsal-, Twi-, and Sna-binding sites, and this element was able to drive marker gene expression in an Apis sim-like pattern (broad lateral stripes) in Drosophila embryos. A comparison of the behavior of the Apis and Drosophila sim enhancers in various mutant and misexpression backgrounds showed that the bee enhancer is extremely sensitive to regulation by Twi and is not responsive to $\mathrm{Su}(\mathrm{H})$, while the opposite is true in both respects for the Drosophila enhancer (Zinzen et al. 2006). These results show that, while the basic logic of sim regulation has been conserved across $\sim 300$ million years of independent evolution (Wiegmann et al. 2009), lineagespecific changes in CRM composition have occurred that may represent adaptations to embryonic patterning environments. So far, it is not known whether the Drosophila or Apis CRM more closely represents the ancestral state, and the resolution of this question will require more indepth sampling of sim regulation throughout the insects. Increased sampling of insect species should also reveal further lineage-specific adaptations of CRMs to the diversity of embryonic patterning environments present in this clade, which will in turn provide further insights into how cis-regulation can change in the face of natural selection.

With the emergence of a large number of fully sequenced insect genomes-along with high-throughput, relatively unbiased techniques (e.g., chromatin immunoprecipita- tion [ChIP] coupled with microarray analysis [ChIP-chip] and ChIP sequencing [ChIP-seq]) to identify DV patterning CRMs-further unexpected themes underlying the evolution of DV patterning control mechanisms have been uncovered. One such discovery was that, despite having no clear homology at the nucleic acid level, CRMs driving DV patterning gene expression are very often located in comparable genomic locations over quite large evolutionary distances (Cande et al. 2009), indicating that CRMs may behave more similarly to exons in the course of evolution than would have been expected. Another discovery was the concept of the "shadow" enhancers, which are elements that often reside quite distant from their targets, but drive similar patterns of expression to already described CRMs. The presence of such redundant elements may provide raw material for evolutionary change without disrupting a running system. Interestingly, the "shadow" enhancer of $b r k$, which is located in an intron of a flanking gene in Drosophila, is conserved in the mosquito Anopheles (Cande et al. 2009), indicating that the position of this enhancer has been conserved through 100 million years of evolution. Thus, it appears that shadow enhancers can be maintained selectively over evolutionary time, and it will be interesting to see whether these redundant enhancers are subject to similar processes that affect duplicated protein-coding genes (e.g., neofunctionalization, subfunctionalization, or nonfunctionalization) (Force et al. 1999).

Post-gastrulation sans Dorsal: patterning the growth zone

Despite the major differences described above, DV patterning at the blastoderm stage in the short-germ embryo of Tribolium is, at its core, basically the same process that patterns the complete embryo of the long-germ fly Drosophila: The blastoderm is essentially a two-dimensional, initially homogenous structure, and the two major axes are patterned perpendicularly and simultaneously make use of maternally generated positional information. The major difference between Tribolium and Drosophila is that, while cells representing the entire future AP axis of the organism receive their DV fate at the blastoderm stage in the Drosophila embryo, only cells representing the future anterior half of the organism are assigned DV fates at this stage in Tribolium, while the rest must be patterned progressively as they emerge from the growth zone (Fig. 4A,B). The growth zone patterning environment differs greatly from that of the blastoderm: The growth zone is a three-dimensional structure composed of cells in differing states of differentiation and cannot rely directly on maternally derived patterning gradients, as the Dorsal gradient has been extinguished by the time the growth zone has formed. Given these differences, the question becomes: How are DV fates assigned progressively to the posterior segments of short-germ embryos?

Disruption of Toll signaling by pRNAi showed that the patterning events occurring at the blastoderm stage are not completely disconnected from those in the growth zone. The mesoderm, marked by Tc-twi expression, is the most ventral fate in Tribolium embryos, and is the fate 
A

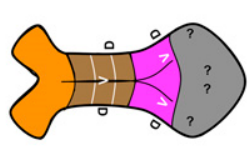

B

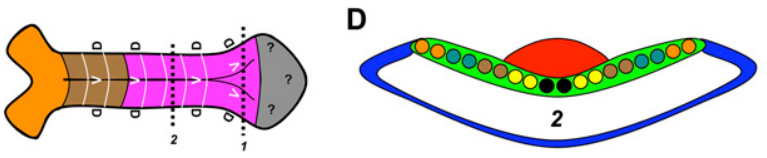

Figure 4. DV patterning of post-gastrulation short-germ embryos. (A) Soon after gastrulation and condensation of the germband, only the most anterior segments have been specified and have clearly established DV cell fates, while the cells in the growth zone that give rise to the majority of the embryo remain uncommitted. (B) As embryogenesis proceeds and cells leave the growth zone, they must simultaneously receive AP and DV positional information to be properly integrated into the extending germband. In $A$ and $B$, orange denotes head, brown denotes thorax, magenta marks abdomen, and gray marks growth zone. (D) Dorsal; (V) ventral. Dashed lines correspond with schematic cross-sections in $C$ and $D .(C)$ Cells just exiting the growth zone encounter two signaling gradients: one with peak levels on the dorsal margin of the germband (BMP signaling; nuclei in shades of red), and one emanating from cells surrounding the ventral midline (MAPK signaling; nuclei in shades of blue). (D) After cells become incorporated into the germband, the signaling gradients were interpreted, and different cell fates along the DV axis were determined (as indicated by different colored nuclei). In $C$ and $D$, red tissue is mesoderm, green is ectoderm, and blue is extraembryonic.

most dependent on Dorsal function at the blastoderm /da Fonseca et al. 2008). The origin of the mesoderm in the growth zone had been unclear because Tc-twi is expressed in two domains in the extending germband: one at the extreme posterior pole of the growth zone, and one in mesenchymal cells in the differentiating segments anterior to it, with the space between devoid of Tc-twi expression (Handel et al. 2005). Thus, it was not clear whether the Tc-twi-expressing mesoderm cells in newly formed segments are derived by induction from the more anterior segments, or whether cells migrate from posterior of the growth zone and reinitiate Tc-twi once they arrive at their proper location. After Tc-Toll pRNAi, the posterior growth zone domain of Tc-twi is unaffected, but both Tc-twi and the mesenchymal cells hypothesized to give rise to the mesoderm are completely absent in the germband. Thus, it appears that all of the mesoderm requires the action of Toll signaling at the blastoderm stage, and mesoderm differentiation in the extending germband is induced from the anterior to the posterior (da Fonseca et al. 2008).

Tc-Toll pRNAi also revealed that DV patterning after gastrulation relies on a self-regulatory patterning system that requires Toll signaling for its proper orientation in relation to the AP axis. Germbands of Tc-Toll pRNAi embryos are not simply dorsalized, but rather show repeated, periodic expression of dorsal markers (dpp and pnr) then lateral ectoderm markers (msh2 and sna). Since these germbands do not receive ventralizing signals, the periodic pattern of expression of different markers likely reflects

a self-organizing property of the DV patterning system in the growth zone that requires Toll function at the blastoderm stage for its proper orientation in relation to the AP axis (da Fonseca et al. 2008).

The self-regulatory aspect of growth zone DV patterning most likely stems from the action of the BMP signaling pathway. When the BMP-binding protein sog /which is expressed at the ventral midline of the germband stage embryol is knocked down with pRNAi, BMP signaling is not simply derepressed. Rather, additional peaks of BMP activation flanking both sides of the ventral midline occur midway between the two normal peaks at the dorsal margins of the germband. The origin of this phenotype is still unknown, but this type of pattern duplication after perturbation is a hallmark of self-regulatory systems (van der Zee et al. 2006).

In addition to the insights from these functional studies, inferences about the mechanism of DV patterning of the ectoderm during germband elongation can be drawn based on the expression of signaling pathway components in combination with knowledge of DV patterning uncovered in Drosophila. While Toll signaling is no longer used after gastrulation in Tribolium (da Fonseca et al. 2008), two other signaling pathways show prominent activation during germband extension: the BMP and EGF signaling pathways. BMP signaling shows high activation at the left and right dorsal flanks of the germband (van der Zee et al. 2006), while EGF activation is strongest in cells directly apposing the ventral midline (Wheeler et al. 2005; Rousso et al. 2010). Based on the presence of these two opposing gradients, it is tempting to propose that cells on either side of the midline integrate signals from both pathways in the process of being specified (Fig. 4C,D).

This idea has a strong basis from what is known in Drosophila, where EGF signaling has ventralizing activity in setting up the expression domains of the columnar genes ( $v n d$, ind, and $m s h$, listed in order of expression from ventral to dorsal), while BMP has a dorsalizing influence (Von Ohlen and Doe 2000). In normal development, the inputs of these pathways into the expression of the columnar genes are largely obscured or made redundant by direct input of Dorsal regulation (Mizutani et al. 2006). Interestingly, among the columnar genes in Tribolium, only vnd is expressed prior to gastrulation, while ind and $m s h$ are expressed medially and laterally, respectively, in the ectoderm of the extending germband (Wheeler et al. 2005). Thus, the domains of expression of these genes are conserved in Tribolium, but the transcriptional inputs driving their expression cannot include Dorsal. Assuming the opposing gradients of BMP and EGF signaling are the sources of DV positional information in the Tribolium germband stage embryo, it appears that, in the Drosophila lineage, Toll signaling has largely usurped the ancestral roles of BMP and EGF signaling in ectodermal patterning in the insects.

The idea of the ventral midline playing a critical ancestral role in patterning the ectoderm of extending germband stage embryos is also supported by recent work in the crustacean Parhyale. When the ventral midline was destroyed by either laser ablation or RNAi against 
sim, the germband ectoderm became completely dorsalized, indicating that the midline is a source of a potent ventralizing activity (Vargas-Vila et al. 2010). The potential roles of the BMP and EGF signaling pathways in ectodermal patterning have not yet been determined in this organism, although a role for sog in providing the midline ventralizing information has been eliminated (Vargas-Vila et al. 2010).

\section{Integrating molecular and classical embryological understandings of insect $D V$ patterning}

Long before the advent of the molecular techniques now commonly used to understand DV patterning in insects, physical manipulations such as fragmentation, cautery, and temperature manipulation of embryos gave many insights into the nature of insect embryogenesis that fit well with our emerging molecular understanding of DV patterning, and suggest that combination of the molecular and classical embryological approaches will allow a deeper understanding of how DV patterning mechanisms can evolve.

One particularly informative set of experiments was the longitudinal fragmentation of embryos of the camel cricket Tachycines. When early germbands were split in half along the ventral midline, these two halves were each able to regulate and give rise to perfectly proportioned twin embryos within the same egg (Krause 1953). When embryos were partially fragmented-for example, where germbands were split only at the anterior-partial axial duplications occurred only in the regions where the two halves of the embryo had been separated. Similar results have been obtained in moths (Krause and Krause 1965) and beetles (Kuethe 1966), among others.

This impressive regulatory capability found in insect germbands is reminiscent of the ability of vertebrate embryos to regenerate well-proportioned embryos or twins upon ligation (De Robertis 2009). Such processes have been found to depend on self-regulatory feedback loops of BMP ligands and inhibitors under opposite transcriptional control (Reversade and De Robertis 2005). Since it has been shown already that BMP signaling has self-regulatory properties in the Tribolium germband embryo (van der Zee et al. 2006), it is tempting to speculate that this property of BMP signaling is used to regenerate the missing halves of the fragmented cricket germbands. Interestingly, while some of the critical factors of the vertebrate BMP self-regulatory system are missing from the Drosophila genome (e.g., BAMBI, ADMP, Noggin, and Gremlin), orthologs of these factors are present in the genomes of more basally branching insect species (Van der Zee et al. 2008; Shigenobu et al. 2010; Werren et al. 2010; JA Lynch, unpubl.). It will be interesting to see whether these genes participate in regulative processes of more basally branching insect species, and whether the loss of BMP signaling components is correlated with a reduced regulative capacity in the lineage leading to Drosophila.

Some insect embryos also display significant regulative capacity prior to the germband stage. For example, longitudinal fragmentation of the leaf hopper Euscelis embryo at the early blastoderm stage leads to the production of fully formed embryos in each egg fragment (Sander 1971). More dramatically, prolonged exposure of embryos of the beetle Atrachya to cold temperatures $\left(\sim 4^{\circ} \mathrm{C}\right)$ at the blastoderm stage leads to the production of up to four complete germbands within a single egg upon reactivation at higher temperatures (Miya and Kobayashi 1974). These results and others like them lead to two conclusions about the evolution of early blastoderm patterning in insects. First, it is clear that, at least in some embryos, maternally derived positional information is not a strict necessity for establishing the DV axis. In the Euscelis case, the orientation of the short axis of the egg with regard to the fragmentation did not affect the production of twinned embryos, as long as both halves received sufficient amounts of posterior pole material and enough cleavage nuclei at the anterior pole. In the case of Atrachya, ectopic germ rudiments condensed randomly with regard to both the $\mathrm{AP}$ and DV axis. In neither case does it seem likely that maternal DV asymmetries play a strong instructive role in patterning the ectopic embryos, leading to the second conclusion that the early blastoderm DV patterning systems in these species should have strong self-regulatory properties that are capable of sensing and amplifying weak asymmetries to give robust patterns in the end. It will be interesting to see if the self-regulation arises from Toll self-regulatory feedback loops such as those found in Tribolium.

\section{The origin of Toll signaling as a DV patterning mechanism}

To date, most of the analyses of the molecular basis of DV patterning evolution of insect embryos has been focused on the regulation and output of the Toll signaling pathway, as this pathway was identified as being the most important source of DV patterning information in Drosophila. However, the use of Toll signaling in the process of DV patterning has been detected only among insects, while this pathway has a highly conserved role in innate immunity throughout the Metazoa. This raises the questions of when in evolution the Toll pathway was recruited for embryonic DV patterning, and what the nature of the ancestral DV patterning system that it replaced was. So far, the use of Toll signaling for DV axis initiation has been demonstrated only in the holometabolous insects Drosophila and Tribolium, but preliminary evidence indicates that it also has an important role in the wasp Nasonia and the bug Oncopeltus (JA Lynch and S Roth, unpubl.). The broad applicability of pRNAi across insect phylogeny, including the most basally branching lineages, should make it relatively straightforward to more precisely map the origin of the role of Toll in DV patterning in this clade.

Results from noninsect arthropods indicate that Toll signaling likely was recruited sometime after the divergence of the insects and their crustacean sister clade. In the crustacean Parhyale, representing the arthropod sister clade of the insects, the embryo forms by holoblastic cleavage, and the fates of the germ layers seem to be determined 
by cell lineage rather than extracellular inductive signaling pathways (Price et al. 2010), indicating that Toll signaling is not used to establish the early DV axis in this organism. In addition, Toll is unlikely to play a key role in patterning the embryo of Achaearaenea, where dpp function is required early to establish both the DV and AP axes, and sog is required for proper DV patterning of the ectoderm later in development (Akiyama-Oda and Oda 2006). The high dependence on the BMP pathway in the spider is suggestive, as this pathway — unlike Toll—has a broadly conserved role in DV axis establishment and patterning throughout the Metazoa. It will be interesting to see if a BMP-based mode of DV patterning was the ancestral state that was later usurped by Toll within insects.

\section{Conclusion}

In order to understand how GRNs change over the course of phylogeny and in response to specific selective challenges, a comparative approach combining detailed descriptions of equivalent GRNs and sampling of taxa at different phylogenetic resolutions will be required. We propose that insects are an ideal evolutionary entity and DV patterning is an ideal developmental process with which to probe the evolution of developmental GRNs.

These practical features of insects as a model clade will allow a broad and deep dissection of questions relating to the evolution of embryonic DV patterning GRNs. Questions of particular interest that remain to be thoroughly addressed include the following: (1) What were the network changes at the cis-regulatory and protein function level required to effect the transition from the ancestral (likely BMP-based) strategy for DV patterning to a patterning mechanism relying primarily on Toll signaling? (2) Are there features of DV GRNs that are characteristic of either long-germ or short-germ embryogenesis? (3) To what extent can the insights gained from studies on DV patterning evolution at the species or genus level be extrapolated to observations made over larger taxonomic distances? (4) How can DV GRNs adapt to respond to novel embryonic patterning environments (i.e., changes in egg shape; radical changes in mode of embryogenesis, such as polyembryony; or novel external environments to which the embryo must adapt, such as high, low, or fluctuating temperatures, or seasonal differences that require embryonic diapause)?

The answers to these and related questions will not only provide insight into the molecular underpinnings of the evolution of DV patterning in insects, but also contribute to a more general understanding of evolutionary developmental mechanisms.

\section{Acknowledgments}

We thank Thomas Buchta, Orhan Özüak, and Dominik Stappert for helpful comments on the manuscript. S.R. and J.A.L. were supported by funding by the SFB 680 .

\section{References}

Akiyama-Oda Y, Oda H. 2006. Axis specification in the spider embryo: dpp is required for radial-to-axial symmetry trans- formation and sog for ventral patterning. Development 133: 2347-2357.

Araujo H, Bier E. 2000. sog and dpp exert opposing maternal functions to modify Toll signaling and pattern the dorsoventral axis of the Drosophila embryo. Development 127: 36313644.

Berghammer AJ, Klingler M, Wimmer EA. 1999. A universal marker for transgenic insects. Nature 402: 370-371.

Brent AE, Yucel G, Small S, Desplan C. 2007. Permissive and instructive anterior patterning rely on mRNA localization in the wasp embryo. Science 315: 1841-1843.

Bucher G, Scholten J, Klingler M. 2002. Parental RNAi in Tribolium (Coleoptera). Curr Biol 12: R85-R86. doi: 10.1016/ S0960-9822(02)00666-8.

Büning J. 1994. The insect ovary. Chapman \& Hall, London.

Cande J, Goltsev Y, Levine MS. 2009. Conservation of enhancer location in divergent insects. Proc Natl Acad Sci 106: 14414 14419.

Chen G, Handel K, Roth S. 2000. The maternal NF-кB/Dorsal gradient of Tribolium castaneum: Dynamics of early dorsoventral patterning in a short-germ beetle. Development 127: 5145-5156.

Cho YS, Stevens LM, Stein D. 2010. Pipe-dependent ventral processing of easter by snake is the defining step in Drosophila embryo DV axis formation. Curr Biol 20: 1133-1137.

Crocker J, Tamori Y, Erives A. 2008. Evolution acts on enhancer organization to fine-tune gradient threshold readouts. PLOS Biol 6: 2576-2587.

da Fonseca RN, von Levetzow C, Kaischeuer P, Basal A, van der Zee M, Roth S. 2008. Self-regulatory circuits in dorsoventral axis formation of the short-germ beetle Tribolium castaneum. Dev Cell 14: 605-615.

da Fonseca RN, van der Zee M, Roth S. 2010. Evolution of extracellular Dpp modulators in insects: The roles of tolloid and twisted-gastrulation in dorsoventral patterning of the Tribolium embryo. Dev Biol 345: 80-93.

Davis GK, Patel NH. 2002. Short, long, and beyond: Molecular and embryological approaches to insect segmentation. Annu Rev Entomol 47: 669-699.

Dearden PK, Akam M. 2001. Early embryo patterning in the grasshopper, Schistocerca gregaria: Wingless, decapentaplegic and caudal expression. Development 128: 3435-3444.

De Robertis EM. 2008. Evo-Devo: Variations on ancestral themes. Cell 132: 185-195.

De Robertis EM. 2009. Spemann's organizer and the selfregulation of embryonic fields. Mech Dev 126: 925-941.

Erives A, Levine M. 2004. Coordinate enhancers share common organizational features in the Drosophila genome. Proc Natl Acad Sci 101: 3851-3856.

Force A, Lynch M, Pickett FB, Amores A, Yan YL, Postlethwait J. 1999. Preservation of duplicate genes by complementary, degenerative mutations. Genetics 151: 1531-1545.

Gabay L, Scholz H, Golembo M, Klaes A, Shilo BZ, Klämbt C. 1996. EGF receptor signaling induces pointed P1 transcription and inactivates Yan protein in the Drosophila embryonic ventral ectoderm. Development 122: 3355-3362.

Handel K, Basal A, Fan X, Roth S. 2005. Tribolium castaneum twist: Gastrulation and mesoderm formation in a short-germ beetle. Dev Genes Evol 215: 13-31.

Hong JW, Hendrix DA, Papatsenko D, Levine MS. 2008. How the Dorsal gradient works: Insights from postgenome technologies. Proc Natl Acad Sci 105: 20072-20076.

Hughes CL, Kaufman TC. 2000. RNAi analysis of Deformed, proboscipedia and Sex combs reduced in the milkweed bug Oncopeltus fasciatus: Novel roles for Hox genes in the Hemipteran head. Development 127: 3683-3694. 
The International Aphid Genomics Consortium. 2010. Genome sequence of the pea aphid Acyrthosiphon pisum. Plos Biology 8: e1000313. doi: 10.1371/journal.pbio.1000313.

Kanodia JS, Rikhy R, Kim Y, Lund VK, DeLotto R, LippincottSchwartz J, Shvartsman SY. 2009. Dynamics of the Dorsal morphogen gradient. Proc Natl Acad Sci 106: 2170721712.

Krause G. 1953. Die Aktionsfolge zur Gestaltung des Keimstreifs von Tachycines (Saltatoria), insbesondere das morphogenetische Konstruktionsbild bei Duplicitas parallela. Dev Genes Evol 146: 275-370.

Krause G, Krause J. 1965. Über das Vermögen median durchschnittener Keimanlagen von Bombyx mori L. sich. in ovo und sich ohne Dottersystem in vitro zwillingsartig zu entwickeln. Z Naturforsch B 20: 334-339.

Kuethe H-W. 1966. Das Differenzierungszentrum als selbstregulierendes Faktorensystem für den Aufbau der Keimanlage im Ei von Dermestes frischi (Coleoptera). Roux'. Archiv fûr Entwicklungsmechanik 157: 212-302.

Liberman LM, Stathopoulos A. 2009. Design flexibility in cisregulatory control of gene expression: Synthetic and comparative evidence. Dev Biol 327: 578-589.

Liberman LM, Reeves GT, Stathopoulos A. 2009. Quantitative imaging of the Dorsal nuclear gradient reveals limitations to threshold-dependent patterning in Drosophila. Proc Natl Acad Sci 106: 22317-22322.

Lynch JA, Desplan C. 2006. A method for parental RNA interference in the wasp Nasonia vitripennis. Nat Protoc 1: 486494.

Lynch JA, Brent AE, Leaf DS, Pultz MA, Desplan C. 2006. Localized maternal orthodenticle patterns anterior and posterior in the long germ wasp Nasonia. Nature 439: 728-732.

Lynch JA, Peel AD, Drechsler A, Averof M, Roth S. 2010. EGF signaling and the origin of axial polarity among the insects. Curr Biol 20: 1042-1047.

Markstein M, Zinzen R, Markstein P, Yee KP, Erives A, Stathopoulos A, Levine M. 2004. A regulatory code for neurogenic gene expression in the Drosophila embryo. Development 131: 2387-2394.

Meinhardt H. 1989. Models for positional signalling with application to the dorsoventral patterning of insects and segregation into different cell types. Development 107: 169-180.

Meinhardt H. 2004. Different strategies for midline formation in bilaterians. Nat Rev Neurosci 5: 502-510.

Miya K and Kobayashi K. 1974. The embryonic development of Atrachya menetriesi. Faldermann (Coleoptera, Chrysomelidae). II. Analyses of early development by ligation and low temperature treatment. J Fac Agric Iwate Univ 12: 39-55.

Miyawaki K, Mito T, Sarashina I, Zhang HJ, Shinmyo Y, Ohuchi H, Noji S. 2004. Involvement of Wingless/Armadillo signaling in the posterior sequential segmentation in the cricket, Gryllus bimaculatus (Orthoptera), as revealed by RNAi analysis. Mech Dev 121: 119-130.

Mizutani CM, Bier E. 2008. EvoD/Vo: The origins of BMP signalling in the neuroectoderm. Nat Rev Genet 9: 663-677.

Mizutani CM, Meyer N, Roelink H, Bier E. 2006. Thresholddependent BMP-mediated repression: A model for a conserved mechanism that patterns the neuroectoderm. PLOS Biol 4: 1777-1788.

Morisato D. 2001. Spatzle regulates the shape of the Dorsal gradient in the Drosophila embryo. Development 128: 23092319.

Moussian B, Roth S. 2005. Dorsoventral axis formation in the Drosophila embryo-Shaping and transducing a morphogen gradient. Curr Biol 15: R887-R899. doi: 10.1016/j.cub.2005. 10.026 .
Nakamura T, Yoshizaki M, Ogawa S, Okamoto H, Shinmyo Y, Bando T, Ohuchi H, Noji S, Mito T. 2010. Imaging of transgenic cricket embryos reveals cell movements consistent with a syncytial patterning mechanism. Curr Biol 20: $1641-1647$.

Netzel H. 1965. Über die Übereinstimmung von Polarität und Bilateralsymmetrie in Embryo, Ei, Oocyte, und Oocytenkern bei Gryllus domesticus L. Roux' Archiv für Entwicklungsmechanik 156: 88-95.

Netzel H. 1968. Die ausprägung von Polarität und Bilateralsymmetrie in den Oocyten von Gryllus domesticus L. Roux' Archiv für Entwicklungsmechanik 160: 119-166.

Neuman-Silberberg FS, Schuepbach T. 1993. The Drosophila dorsoventral patterning gene gurken produces a dorsally localized RNA and encodes a TGF- $\alpha$-like protein. Cell 75: 165-174.

O'Connor MB, Umulis D, Othmer HG, Blair SS. 2006. Shaping BMP morphogen gradients in the Drosophila embryo and pupal wing. Development 133: 183-193.

Ohde T, Masumota M, Yaginuma T, Niimi T. 2009. Embryonic RNAi analysis in the firebrat, Thermobia domestica: Distalless is required to form caudal filament. I Insect Biotechnol Sericology 78: 99-105.

Olesnicky EC, Desplan C. 2007. Distinct mechanisms for mRNA localization during embryonic axis specification in the wasp Nasonia. Dev Biol 306: 134-142.

Papatsenko D. 2007. ClusterDraw web server: A tool to identify and visualize clusters of binding motifs for transcription factors. Bioinformatics 23: 1032-1034.

Pavlopoulos A, Berghammer AJ, Averof M, Klingler M. 2004. Efficient transformation of the beetle Tribolium castaneum using the Minos transposable element: Quantitative and qualitative analysis of genomic integration events. Genetics 167: 737-746.

Peloquin JI, Thibault ST, Staten R, Miller TA. 2000. Germ-line transformation of pink bollworm (Lepidoptera: gelechiidae) mediated by the piggyBac transposable element. Insect Mol Biol 9: 323-333.

Petersen CP, Reddien PW. 2009. Wnt signaling and the polarity of the primary body axis. Cell 139: 1056-1068.

Price AL, Modrell MS, Hannibal RL, Patel NH. 2010. Mesoderm and ectoderm lineages in the crustacean Parhyale hawaiensis display intra-germ layer compensation. Dev Biol 341: 256-266.

Reversade B, De Robertis EM. 2005. Regulation of ADMP and BMP2/4/7 at opposite embryonic poles generates a selfregulating morphogenetic field. Cell 123: 1147-1160.

Richards S, Gibbs RA, Weinstock GM, Brown SJ, Denell R, Beeman RW, Gibbs R, Bucher G, Friedrich M, Grimmelikhuijzen CJP, et al. 2008. The genome of the model beetle and pest Tribolium castaneum. Nature 452: 949-955.

Roth S. 2003. The origin of dorsoventral polarity in Drosophila. Philos Trans R Soc Lond B Biol Sci 358: 1317-1329.

Roth S. 2004. Gastrulation in other insects. In Gastrulation (ed. C. Stern), pp. 105-121. Cold Spring Harbor Laboratory Press, Cold Spring Harbor, NY.

Roth S, Lynch JA. 2009. Symmetry breaking during Drosophila oogenesis. Cold Spring Harb Perspect Biol 1: a001891. doi: 10.1101/cshperspect.a001891.

Roth S, Schupbach T. 1994. The relationship between ovarian and embryonic dorsoventral patterning in Drosophila. Development 120: 2245-2257.

Rousso T, Lynch J, Yogev S, Roth S, Schejter ED, Shilo BZ. 2010. Generation of distinct signaling modes via diversification of the Egfr ligand-processing cassette. Development 137: 34273437. 
Sander K. 1971. Pattern formation in longitudinal halves of leaf hopper eggs (Homoptera) and some remarks on definition of embryonic regulation. Wilhelm Roux' Arch Entwickl Mech Org 167: 336.

Sandmann T, Girardot C, Brehme M, Tongprasit W, Stolc V, Furlong EE. 2007. A core transcriptional network for early mesoderm development in Drosophila melanogaster. Genes \& Dev 21: 436-449.

Schupbach T, Roth S. 1994. Dorsoventral patterning in Drosophila oogenesis. Curr Opin Genet Dev 4: 502-507.

Schweitzer R, Shaharabany M, Seger R, Shilo BZ. 1995. Secreted Spitz triggers the DER signaling pathway and is a limiting component in embryonic ventral ectoderm determination. Genes Dev 9: 1518-1529.

Sen J, Goltz JS, Stevens L, Stein D. 1998. Spatially restricted expression of pipe in the Drosophila egg chamber defines embryonic dorsal-ventral polarity. Cell 95: 471-481.

Shigenobu S, Bickel RD, Brisson JA, Butts T, Chang CC, Christiaens O, Davis GK, Duncan EJ, Ferrier DEK, Iga M, et al. 2010. Comprehensive survey of developmental genes in the pea aphid, Acyrthosiphon pisum: Frequent lineage-specific duplications and losses of developmental genes. Insect Mol Biol 19: 47-62.

Stathopoulos A, Levine M. 2004. Whole-genome analysis of Drosophila gastrulation. Curr Opin Genet Dev 14: 477-484.

Stathopoulos A, Levine M. 2005. Genomic regulatory networks and animal development. Dev Cell 9: 449-462.

Tamura T, Thibert C, Royer C, Kanda T, Abraham E, Kamba M, Komoto N, Thomas JL, Mauchamp B, Chavancy G, et al. 2000. Germline transformation of the silkworm Bombyx mori L. using a piggyBac transposon-derived vector. Nat Biotechnol 18: $81-84$.

van der Zee M, Stockhammer O, von Levetzow C, da Fonseca RN, Roth S. 2006. Sog/Chordin is required for ventral-todorsal Dpp/BMP transport and head formation in a short germ insect. Proc Natl Acad Sci 103: 16307-16312.

Van der Zee M, da Fonseca RN, Roth S. 2008. TGF $\beta$ signaling in Tribolium: Vertebrate-like components in a beetle. Dev Genes Evol 218: 203-213.

Vargas-Vila MA, Hannibal RL, Parchem RJ, Liu PZ, Patel NH. 2010. A prominent requirement for single-minded and the ventral midline in patterning the dorsoventral axis of the crustacean Parhyale hawaiensis. Development 137: 34693476.

Von Ohlen T, Doe CQ. 2000. Convergence of dorsal, Dpp, and Egfr signaling pathways subdivides the Drosophila neuroectoderm into three dorsal-ventral columns. Dev Biol 224: 362-372.

Wang YC, Ferguson EL. 2005. Spatial bistability of Dpp-receptor interactions during Drosophila dorsal-ventral patterning. Nature 434: 229-234.

Weinstock GM, Robinson GE, Gibbs RA, Worley KC, Evans JD, Maleszka R, Robertson HM, Weaver DB, Beye M, Bork P, et al. 2006. Insights into social insects from the genome of the honeybee Apis mellifera. Nature 443: 931-949.

Werren JH, Richards S, Desjardins CA, Niehuis O, Gadau J, Colbourne JK, Beukeboom LW, Desplan C, Elsik CG, Grimmelikhuijzen CJP, et al. 2010. Functional and evolutionary insights from the genomes of three parasitoid Nasonia species. Science 327: 343-348.

Wheeler SR, Carrico ML, Wilson BA, Skeath JB. 2005. The Tribolium columnar genes reveal conservation and plasticity in neural precursor patterning along the embryonic dorsalventral axis. Dev Biol 279: 491-500.

Wiegmann BM, Trautwein MD, Kim JW, Cassel BK, Bertone MA, Winterton SL, Yeates DK. 2009. Single-copy nuclear genes resolve the phylogeny of the holometabolous insects. BMC Biol 7: 34. doi: 10.1186/1741-7007-7-34.

Zeitlinger J, Zinzen RP, Stark A, Kellis M, Zhang H, Young RA, Levine M. 2007. Whole-genome ChIP-chip analysis of Dorsal, Twist, and Snail suggests integration of diverse patterning processes in the Drosophila embryo. Genes Dev 21: 385-390.

Zhang ZY, Stevens LM, Stein D. 2009a. Sulfation of eggshell components by pipe defines dorsal-ventral polarity in the Drosophila embryo. Curr Biol 19: 1200-1205.

Zhang ZY, Zhu XI, Stevens LM, Stein D. 2009b. Distinct functional specificities are associated with protein isoforms encoded by the Drosophila dorsal-ventral patterning gene pipe. Development 136: 2779-2789.

Zinzen RP, Cande J, Ronshaugen M, Papatsenko D, Levine M. 2006. Evolution of the ventral midline in insect embryos. Dev Cell 11: 895-902. 


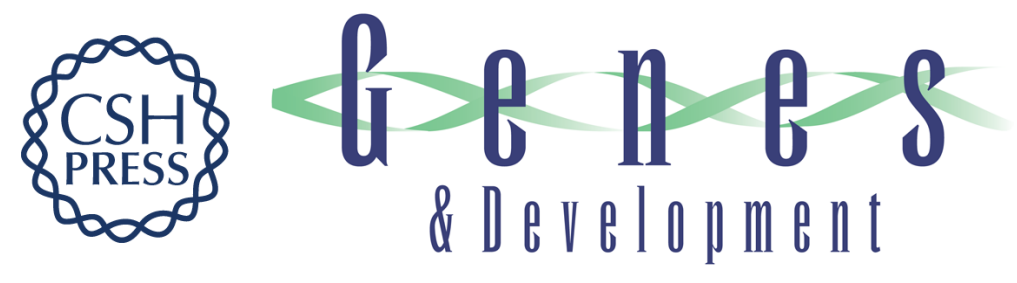

\section{The evolution of dorsal-ventral patterning mechanisms in insects}

Jeremy A. Lynch and Siegfried Roth

Genes Dev. 2011, 25:

Access the most recent version at doi:10.1101/gad.2010711

References This article cites 80 articles, 26 of which can be accessed free at: http://genesdev.cshlp.org/content/25/2/107.full.html\#ref-list-1

License

Email Alerting Receive free email alerts when new articles cite this article - sign up in the box at the top Service right corner of the article or click here.

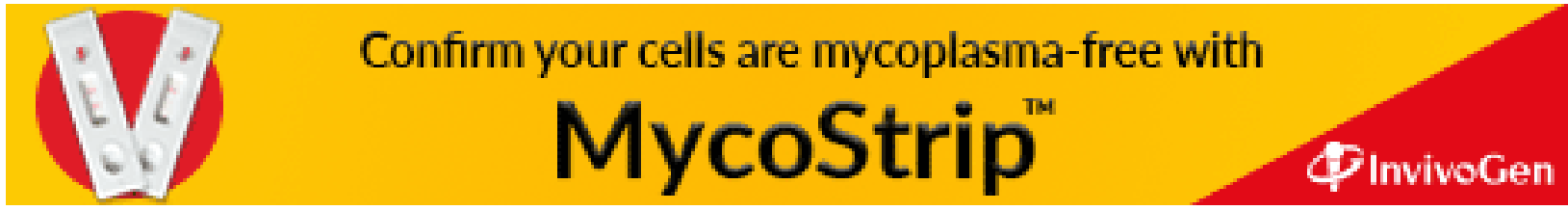

